\title{
Increase the photostability of aqueous dispersed QDs using surfactants as stabilizing agent under long- time UV-light irradiation
}

\begin{abstract}
Luminescent semiconductor quantum dots (QDs) have ideal optical properties and have been used to tag biomolecules for bio-detection and bio-imaging. Still today it is an argue about the life-time of the photoluminescence of monodispersed aqueous quantum dots (MAQD). Effort was taken to improve the photoluminescence life-time of monodispersed aqueous quantum dots (MAQD) using different surface fictionalization agents during the synthesis process. In this paper, first time we present a comparative stability increase of aqueous dispersed semiconductor ((CdSe) $\mathrm{ZnSe}) \mathrm{ZnS}$ quantum dots followed by the increase of photoluminescence life-time using Sodiumdodecyl sulfate(SDS), Cetyltrimethylammonium bromide(CTAB), Sodiumdodecylbebzene sulfunate(SDBS) surfactants as stabilizing agent.
\end{abstract}

Volume 4 Issue 3 - 2018

\author{
Abu Mohammad Azmal Morshed \\ Department of Chemistry, Bangladesh University of Textiles \\ (BUTEX), Bangladesh \\ Correspondence: Abu Mohammad Azmal Morshed, \\ Department of Chemistry, Bangladesh University of Textiles \\ (BUTEX), Dhaka- I208, Bangladesh, \\ Email azmal.morshed@gmail.com
}

Received: February 25, 2018 | Published: May 17, 2018

Keywords: luminescence, monodispersed, quantum dots, fictionalization

\section{Introduction}

Quantum dots (QDs) have attracted a great deal of attention over the past two decades because of their unique photochemical and photo physical properties. Colloidal semiconductor quantum dots (QDs) have great potential as a new class of fluorophores for biological and biomedical imaging because of high brightness, long-term photostability and single-light source excitation for multicolored QDs. ${ }^{1,2}$ The syntheses of monodispersed fluorescent QDs are generally performed in organic solvents with surface passivation by alkyl phosphine oxides such as trioctylphosphine oxide (TOPO). ${ }^{3,4}$ The resulting QDs are soluble only in nonpolar solvents that making them difficult to use for biological application. So far, many synthetic methodologies for the preparation of water-soluble QDs have been developed by surface modifications with amphiphilic compounds including thiols ${ }^{1,2,5-8}$ polymers, ${ }^{9,10}$ phpholipids. ${ }^{11}$ In recent years, there has been great progress in synthetic methods of quantum dot production, which made large scale preparation of QDs with high quality and narrow size distribution possible. Luminescent QDs have been successfully attached to protein, sugar, and other biologically active agents. ${ }^{9,12-14}$ Among the various types of modified QDs have been studied most thoroughly due to their possible applications in biological disciplines. However, consideration of QDs behavior at biological interfaces, e.g., cell membranes, is one of the most important issues requiring further study prior to implementation in real applications. Currently, few studies have been conducted regarding surface chemistry properties of quantum dots at biological interfaces. Quantum dots synthesized in organic solvents are insoluble in water. Hence a challenge is how to make the highquality hydrophobic quantum dots soluble in water and also active in bioconjugate reactions. Ligand exchange inevitably alters the chemical and physical states of the quantum dot surface atoms and in most cases dramatically decreases the quantum efficiency of the quantum dots, thiol-based molecules (e.g.,mercaptocarbonic acids) may form disulfides over time and come off from the quantum dot surface and finally the quantum dots aggregate and precipitate out of water. Surface functionalization of quantum dots can improve the solubility but reduce the quantum efficiency.

Our aim is to improve both the properties as: (1). Improve the solubility of quantum dots,(2) Stability in aqueous dispersion(3) Retain the quantum efficiency followed by to reduce the toxicity of quantum yield for biological cell labeling. Standard methods for water-solubilizing of quantum dots involve conjugating them to polar molecules containing thiol groups or embedding them in the nonpolar region of micelles or vesicles. Unfortunately, thiol compounds are notorious for their quenching of nanocrystal emission and precipitation under continuous light exposure. They do, however, preserve the initial nanocrystal size and provide an intermediate to which other molecules, such as proteins and nucleic acids, can be attached. Few authors tried the surface functionalization with surfactants during synthesis process of quantum dots nanocrystals. It has been established that in air the oxygen molecule oxidized chalcogenide atoms as Se, S located at the surface of quantum dots to form $\mathrm{SeO}_{2}$ and $\mathrm{SO} 42-$. In this case $\mathrm{CdSe}$ quantum dots $\mathrm{SeO}_{2}$ molecule absorbs and leaving behind "dangling" reduced $\mathrm{Cd}$-ions, $\mathrm{Cd}$ atoms. This prolonged espouser to of quantum dots to an oxidative environment can decomposition of the CdSe nanocrystal therefore leading to decomposition $\mathrm{Cd}$ ion or CdSe complex from the core. In our work we used simple surface functionalization of TOPO capped quantum dots during solublization process to increase the disperse stability thus to give fluorescence life time. In the current study, ((CdSe) $\mathrm{ZnSe})) \mathrm{ZnS}$ quantum dots have been modified with SDS, CTAB, SDBS surfactants. QD-surfactants can form a long time stable monolayer at air/water interface.

\section{Experimental section}

\section{Chemicals}

TOPO capped ((CdSe)ZnSe))ZnS quantum dots was purchased from Nanosquare Company Ltd. Seoul, Korea. Sodiumdodecyl sulfate (SDS), Cetyltrimethylammonium bromide (CTAB), Sodiumdodecylbebzene sulfunate(SDBS) were Sigma brand at 99 
$\%$ purity. Marceptoacetic acid (MAA) from TCI, Japan (99\%). All organic solvents were used from Aldrich Co. All chemicals were directly used in experimental preparations without further purification.

\section{Preparation aqueous dispersed ((CdSe)ZnSe)ZnS} quantum dots

$((\mathrm{CdSe}) \mathrm{ZnSe})) \mathrm{ZnS}$ was solubilized using Mercaptoacetic acid (MAA), which replaces the TOPO coating allowing the quantum dots to be suspended in aqueous solution. ${ }^{15,16}$ Briefly, $0.50 \mathrm{~mL}$ of MAA was added to $0.05 \%$ wt TOPO-capped ((CdSe)ZnSe $)) \mathrm{ZnS}$ QDs in Chloroform and rocked gently for $2 \mathrm{~h}$ in the dark. ${ }^{17}$ Then $20 \mathrm{ml}$ of distilled water was added into the QDs in Chloroform solution. The solution was vigorously agitated, and the layers were allowed to separate naturally. The pigmented layer was removed and centrifuged for $5 \mathrm{~min}$ at $5000 \mathrm{rpm}$ in a micro centrifuge. Then the supernatant was separated and was stored in a container. $\mathrm{pH}$ of the solution was measured about 2.4 ( $\mathrm{pH}$ measurement was done using a $\mathrm{pH}$ meter of Thermo electronic corporation model $420 \mathrm{~A}+$ ). Finally, the resulting colloid was stored in refrigerator for the further experiment. All the procedure was carried out in dark condition to prevent photo degradation reaction of quantum dots.

\section{Original concentration measurement in QD-MAA solution}

As we used $0.05 \% \mathrm{w} / \mathrm{v}$ QDs first solublized in Chloroform and finally in aqueous with MAA. During the whole process i.e., Centrifugation and decanting the solution there is loss of quantum dots from its total amount $(0.05 \% \mathrm{wt})$. The real concentration of QDs in QD-MAA solution was determined from absorbance difference (Figure 1) in UV visible spectrophotometer ${ }^{18}$ from Beers- Lambert equation:

$$
A=\varepsilon b c
$$

And was found that the real concentration of QDs in aqueous MAA solution was $0.04 \% \mathrm{wt}$. And a total amount of $0.01 \% \mathrm{wt}$ of quantum dot was lost during solution preparation processing (Centrifugation, phase separation, decanting etc).

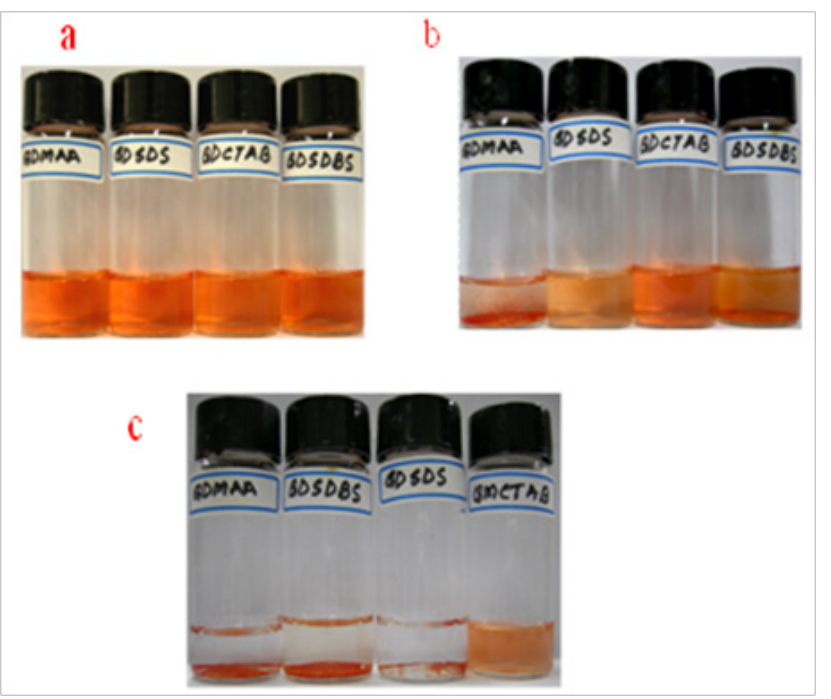

Figure I Visual changes of QDs aqueous dispersed solution under UV-light irradiation

\section{Photostability analysis}

Prolonged UV exposure causes two possible processes to occur with $(((\mathrm{CdSe}) \mathrm{ZnSe}) \mathrm{ZnS})$ quantum dots water-solubilized with thiol compounds: (1) Decay of the thiol coat through the formation of disulfide bonds, which leads to $(((\mathrm{CdSe}) \mathrm{ZnSe}) \mathrm{ZnS})$ precipitation, and (2) Possible coordination of surfactant molecules to the (((CdSe) $\mathrm{ZnSe}) \mathrm{ZnS}$ )surface, which creates long-lived surface trap states and enhanced (( (CdSe)ZnSe)ZnS)exciton emission. The first process helps the second process by increasing surface exposure to the environment. Thus causes the loss of solubility and aggregation of $(((\mathrm{CdSe}) \mathrm{ZnSe})$ ZnS)MAA over time. All photooxidation experiments were performed in a photooxidation chamber under constant airflow with a SCINCO UV -Spectrophotometer model S-2030 multiband Ultraviolet lamp. The lamp was set to $365 \mathrm{~nm}$ (short wavelength) and was placed $4 \mathrm{~cm}$ directly above the samples. The samples were placed in $1-\mathrm{cm}$ polypropylene cells, and the sample volume was $\sim 3 \mathrm{~mL}$. The samples were prepared by adjusting the absorbance (optical density, OD) of the purified nanocrystals at the first excitation absorption peak to 0.50.3 . The UV-visible absorption spectra were recorded at certain time (hours) intervals.

\section{Optical density (OD) measurement}

Both the aqua-QDs-MAA and aqua-QDs-MAA-surfactants solution were kept under UV-light (condition described above) during the total experimental time. The aliquot was taken out from the total solution after different time intervals. Visual photo was taken by a Nikon- complex P2 camera, Nikon Corporation, Japan. UVVisible absorption spectra of aqua-QDs-MAA and aqua-QDs-MAASurfactants were recorded by a CAYR-100 HP spectrophotometer using $1 \mathrm{~cm}$ quartz cells.

\section{Results}

\section{Stability measurement}

The photochemical instability of thiolcoated ((CdSe)ZnSe)ZnS)) nanocrstyals can be readily monitored by recording the UV-vis spectra of the samples dissolved in aqua-surfactants media at different time intervals. The size-dependent absorption of ((CdSe)ZnSe) ZnS) nanocrystals provides a sensitive and convenient detection probe of the photooxidation of the nanocrystals themselves. As shown in Figure 1, upon the photooxidation of $((\mathrm{CdSe}) \mathrm{ZnSe}) \mathrm{ZnS})$ nanocrystals, the size of nanocrystals became smaller, and the peak position due to the first exciton absorption of nanocrystals shifted. At the same time, the absorbance or optical density (OD) at the original absorption peak decreased also due to the shrinkage of the nanocrystals. The precipitation of the nanocrystals caused the obvious nonzero baseline for the last spectrum in Figure 1. Thiols with a low molecular mass sometimes precipitated out of the solution by showing none or very few nanometers of peak shift shown in Figure 3 and summarized in Table 2.

Therefore, the temporal evolution of the optical density was a more accurate indicator for the photooxidation processes in most cases. In the oxidative environment a progressive change in the color and absorbance profile of the quantum dot solution was observed. Aqueous stability was compared from UV-visible spectroscopy study comparing the peak intensity of QD-aqueous with QD-micelles with time. ${ }^{18}$ As the amount of $\mathrm{Cd}$ reduced by oxidation the peak height reduces as we know peak height is related with QD concentration ${ }^{15}$ 
shown in Figure 2 and the shifting of peak position summarized in Table 1.and visual comparison in Figure 1. From our study we found that aqueous dispersed quantum dots can be made 200 times more stable using CTAB micelles then only in water. Our next work is to find out better stabilizing agent as surface coating agent for quantum dots that can make QDs aqueous dispersion more stable for long time photostability in biological labeling.

Table I Peak position shift as the oxidation progress with time

\begin{tabular}{lllll}
\hline Time & \multicolumn{4}{l}{ Names of functionalizing agents } \\
\hline & MAA & SDS & CTAB & SDBS \\
0.0000 & 561.5000 & 562.1500 & 563.5600 & 562.1500 \\
3.0000 & 562.0000 & 563.1100 & 562.0000 & 563.1100 \\
18.0000 & 562.0000 & 565.0400 & 561.0000 & 566.9600 \\
30.0000 & 563.5000 & 566.4800 & 560.8100 & 568.8900 \\
44.0000 & 564.0000 & 567.9300 & 559.7500 & 570.2000 \\
50.0000 & 564.9600 & 569.0000 & 558.3400 & 570.8200 \\
65.0000 & 565.3400 & 569.7300 & 557.0000 & 571.7500 \\
90.0000 & - & 570.3800 & 556.5000 & 571.9600 \\
138.0000 & - & - & 556.0000 & 572.7500 \\
160.0000 & - & - & 555.6400 & - \\
184.0000 & - & - & 554.0000 & - \\
\hline
\end{tabular}
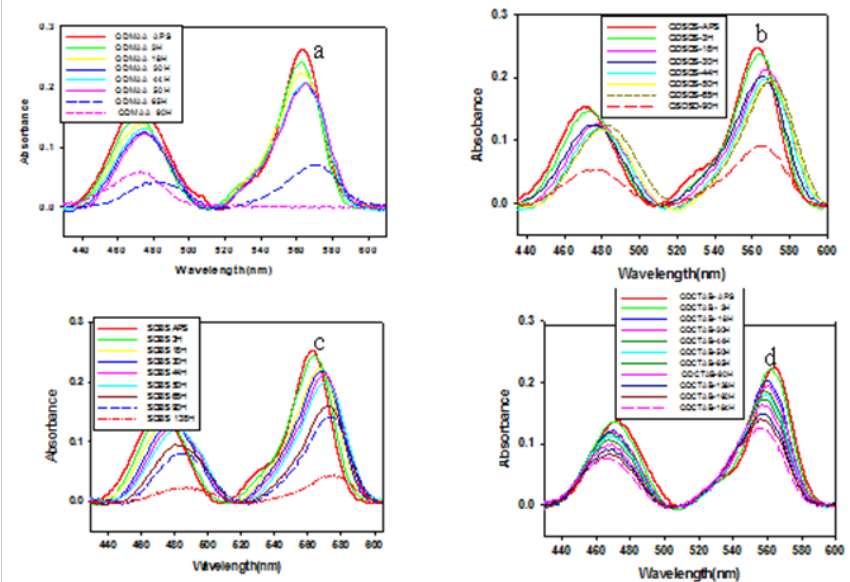

Figure 2 UV- visible spectra of QDs aqueous dispersed solution (A) QDs only with MAA,

(B) QDs with MAA and SDS, (C) QDs MAA and SDBS and (D) QDs with $M A A$ and $C T A B$.

Table 2 Change of peak height (maximum absorbance) as the oxidation progress with time

\begin{tabular}{|c|c|c|c|c|}
\hline \multirow[t]{2}{*}{ Time } & \multicolumn{4}{|c|}{ Names of functionalizing agents } \\
\hline & MAA & SDS & CTAB & SDBS \\
\hline 0.0000 & 0.3395 & $0.247 \mid$ & 0.2244 & 0.2524 \\
\hline 3.0000 & 0.3061 & 0.2404 & 0.2231 & 0.2438 \\
\hline 18.0000 & 0.2859 & 0.2088 & 0.2020 & 0.2210 \\
\hline 30.0000 & 0.2773 & 0.2011 & 0.1940 & 0.2181 \\
\hline 44.0000 & 0.2697 & 0.1998 & 0.1856 & 0.2125 \\
\hline 50.0000 & 0.2678 & 0.1957 & 0.1786 & 0.1994 \\
\hline 65.0000 & 0.1157 & 0.1943 & 0.1726 & 0.1594 \\
\hline 90.0000 & - & 0.0987 & 0.1635 & 0.1420 \\
\hline 138.0000 & - & - & 0.1400 & 0.0428 \\
\hline 160.0000 & - & - & 0.1246 & - \\
\hline 184.0000 & - & - & 0.1096 & - \\
\hline
\end{tabular}

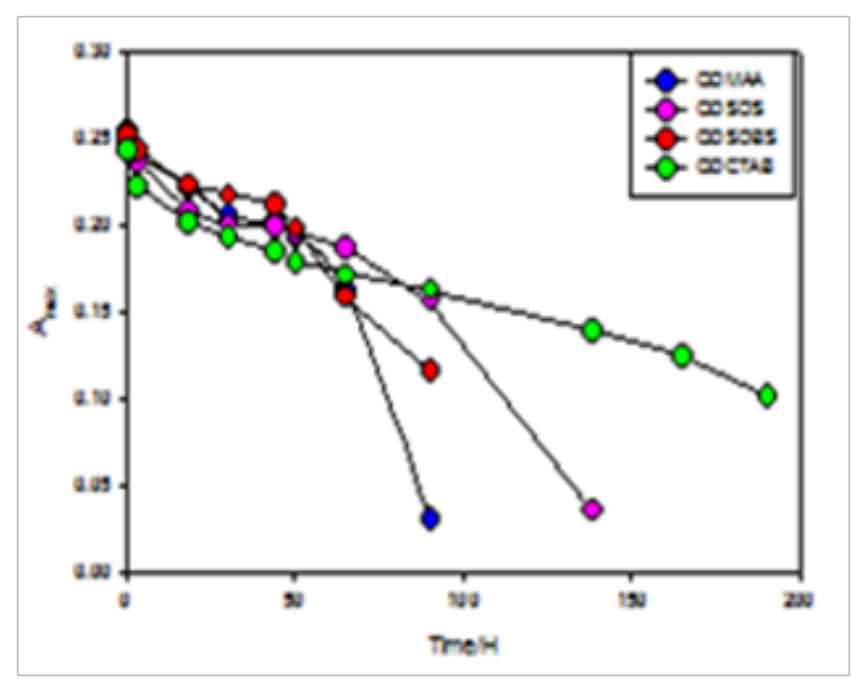

Figure 3 Decreased peak intensity with time as the result of progress oxidation under UV-light irradiation.

\section{Comparative stability assessment among different surfactants}

Luminescence lifetime and dispersed stability was observed under UV-light irradiation from visible changes and the decreased intensity of UV-visible absorption spectra. It was found that the aqueous dispersed quantum dots ((CdSe)ZnSe)ZnS only with MAA was dispersed only for 55 hours after preparation under the UV-light experimental condition. As we used stabilizing agents i.e., surface functionalizing agents SDS, SDBS and CTAB then the dispersed stability and the luminescence lifetime increased comparatively in the order $(((\mathrm{CdSe})$ $\mathrm{ZnSe}) \mathrm{ZnS})-\mathrm{MAA}<(((\mathrm{CdSe}) \mathrm{ZnSe}) \mathrm{ZnS})-\mathrm{MAA}) \quad \mathrm{SDS}<(((\mathrm{CdSe}) \mathrm{ZnSe})$ $\mathrm{ZnS})-\mathrm{MAA})$ SDBS $<(((\mathrm{CdSe}) \mathrm{ZnSe}) \mathrm{ZnS})$ MAA $)-\mathrm{CTAB}$. So in our experimental observation we found that using SDS, SDBS and CTAB as stabilizing agent for $((\mathrm{CdSe}) \mathrm{ZnSe}) \mathrm{ZnS}$ quantum dots can improve luminescence lifetime $40 \%, 66 \%$ and $73 \%$ respectively as compared than $((\mathrm{CdSe}) \mathrm{ZnSe}) \mathrm{ZnS})$ quantum dots aqueous solution only with MAA

\section{Discussion}

QD-micelle solutions exhibited the same visible and emission colors as hydrophobic QDs, as shown in the optical micrograph (Figure 1). The formation of individual ((CdSe)ZnSe)ZnS) QDmicelles was further characterized by UV-visible spectroscopy shown in Figure 2. We observed no difference in the position or width of the absorbance bands at $\sim 454 \mathrm{~nm}$ and $\sim 563 \mathrm{~nm}$ from hydrophobic QDs and QD-micelles, which suggests that QD-micelles maintain their optical properties in water. ${ }^{19}$ It was observed (((CdSe)ZnSe)ZnS)-MAA) aqueous dispersion was stable up to 55 hours of after preparation but (((CdSe)ZnSe)ZnS)-MAA)-micelles aqueous dispersion was $\sim 160 \mathrm{~h}$ stable shown by visual picture and also by UV-visible measurement in Figure 1. The loss of solubility and aggregation of $((\mathrm{CdSe}) \mathrm{ZnSe})$ ZnS)-MAA) over time was followed spectrophotometrically with the absorption spectra shown in the Figure 1. Studies of photostability of the water-soluble QD- micelles under long-time UV-light showed that the QD-micelles exhibited comparatively less loss of UV-visible peak height than that of in QD-water solution Figure 1. 
The results revealed that the photochemical instability of the nanocrystals actually included three distinguishable processes, namely the photocatalytic oxidation of the thiol ligands on the surface of nanocrystals, the photooxidation of the nanocrystals, and the precipitation of the nanocrystals. At first, the thiol ligands on the surface of a nanocrystal were gradually photo catalytically oxidized using the CdSe nanocrystal core as the photocatalyst. This photocatalytic oxidation process was observed as a zero-order reaction in terms of the concentration of the free thiols in the solution. The photogenerated holes in a nanocrystal were trapped onto the thiol ligands bound on the surface of the nanocrystal, which initiated the photooxidation of the ligands and protected the nanocrystal from any photooxidation. After nearly all of the thiol ligands on the surface of the nanocrystals were converted into disulfides, the system underwent several different pathways. If the disulfides were soluble in water, then all of the disulfides fell into the solution at the end of this initial process, and the nanocrystals precipitated out of the solution without much variation over their size and size distribution. When the disulfides were insoluble in water, they likely formed a micelle-like structure around the nanocrystal core and kept it soluble in the solution. In this case, the nanocrystals only precipitated after severe oxidation, which took a long period of time. If the system contained excess free thiol ligands, they replaced the photochemically generated disulfides and maintained the stability and solubility of the nanocrystals. The initiation stage of the photooxidation of $(((\mathrm{CdSe})$ $\mathrm{ZnSe}) \mathrm{ZnS}$ ) nanocrystals themselves increased as the thickness and packing density of the ligand shell increased. This was explained by considering the ligand shell on the surface of a nanocrystal as the diffusion barrier of the oxygen species from the bulk solution into the interface between the nanocrystal and the surface ligands. ${ }^{20}$

Experimental results clearly indicated that the initiation stage of the photooxidation was not caused by the chemical oxidation of the system kept in air under dark conditions or the hydrolysis of the cadmium-thiol bonds on the surface of the nanocrystals, both of which were magnitudes slower than the photocatalytic oxidation of the surface ligands if they occurred at all. The advantage of our method to provide water-soluble QD-micelles is its capability to utilize a wide variety of surfactants/lipids with different functionally terminated groups, which can be used to improve biocompatibility and to enable bioconjugation. In our method, the stabilization of hydrophobic QDs in water relies on the thermodynamically favorable interdigitation of surfactants and QD stabilizing ligands formed after solvent evaporation, enabling rapid efficient transfer of the QDs into the aqueous phase. The QD micelles preserve the optical properties of the original source QDs, such as absorbance value and lifetime etc., as the encapsulation process involves no chemical substitution reactions. In contrast, in other methods, such as the ligand exchange method, chemical substitution occurs at the QD interface.

This usually results in QD aggregation and changes in QD physical properties. ${ }^{19,20}$ Both air and UV light have been reported to induce and catalyze, respectively, the oxidation of nanoparticle surfaces. ${ }^{15,21}$ After exposure to an oxidative environment, we observed a progressive change in the color and absorbance profile of the QD solution UVvisible absorption peak, and a decrease in the quantum yield (Figures 2) (Table 1). These results are in agreement with previous oxidation studies. ${ }^{22}$ Shifts in the absorbance (Figures 3 ) (Table 2) spectra occur because of a decrease in the size of the nanoparticle. Oxygen attacks exposed Se sites, resulting in the desorption of $\mathrm{SeO}_{2}$ and a reduction in the nanocrystal diameter with a concurrent shift of the emission spectrum to the UV. The shift of spectra occurs because of the decrease of the size of nanoparticles, loss of surface atoms due to oxidation. Red shift in the UV-visible absorption peak is due to the formation of lower energy band gaps induced by newly formed defects. In our observation during the use of different surface functionalizing agent SDS, SDBS and CTAB, we find different observation in the peak position shift. In case of SDS and SDBS the peak position shift is in same direction at the red region but opposite in case of CTAB which give more stability of aqueous dispersed ((CdSe) $\mathrm{ZnSe}) \mathrm{ZnS})$ ) quantum dots. The peak position shift is toward the green region could be the result of ionic environment around the quantum dots nanocluster and/ or physical oxidation with dissolved $\mathrm{O}_{2}$ of the exposed nanocluster surface resulting the disorbtion of $\mathrm{SeO}_{2}$ and smaller CdSe core cells. Finally, the corresponding red shift and broadening of the steady-state emission spectrum of $((\mathrm{CdSe}) \mathrm{ZnSe}) \mathrm{ZnS}$ is probably due to increased particle diameter and less heterogeneous size distribution of the aqueous dispersed QDs.

With the increase of surface oxidation at the air water interface of quantum dots aqueous dispersion the change of peak width is remarkable shown in Figure 4 and values are summarized in Table 3, which can be described relating with the decreased band gap energy of quantum dots core cells CdSe with the resulting oxidation at air/ water interface. Still there is not enough discussion on the change of peak width in UV-visible spectra with the progress of oxidation. So the fact could be depending probably on the Van-der-Wall force of attraction between the quantum dots outer surface atoms and the thiol group. As the oxidation progresses with time producing disulfide of sulphide of thiol groups of MAA than the percentage of thiol group start to reduce from the surface of the quantum dots thus produce holes between the contentious surrounding of thiol groups. So the QDs core cell CdSe comes in contact of photo light which is very sensitive to photo reduction by cause of oxidation resulting $\mathrm{Cd}^{2+}$ and $\mathrm{SeO}_{2}$ surface. Progress of the oxidation process effect the quantum dots QDs dispersed environment is as: loss the number of surface binding functional group (thiols) causes the oxidation of the core cells of quantum dots reducing the size of quantum dots. Continuous oxidation keeps on decreasing the thiol groups increase the hole gape to enter more photos light ultimately causes increase in the oxidation rate of QDs core cells. As a result the bond energy ( Wan der waals force of attraction) at the surface of QDs looses and in continuous decreasing the binding energy causes increase in the total area of nanocluster(NC) and finally breaks the bond between the surface functionalized molecules and the QDs and then the QDs falls off out of the solution as precipitate The increase size of peak width is due to the increase in total surface area of QDs along with the functionalized molecule and the decrease of peak intensity is the result of reduction of $\mathrm{Cd}$ atom as $\mathrm{Cd}^{2+}$ ion as a result of color degradation (Figure 1).

In a similar manner, we would expect the addition of surface coatings (DHLA $,{ }^{19} \mathrm{BSA},{ }^{9}$ polyacrylate ${ }^{23}$ ), typically added to increase the quantum yield and stability of nanoparticles, to decrease the surface oxidation. To test the effects of surface coatings on the stability of aqueous dispersed QDs several anionic (SDS, SDBS), cationic(CTAB) surfactants $(0.5 \% \mathrm{wt})$ were added with $((\mathrm{CdSe})$ $\mathrm{ZnSe}) \mathrm{ZnS})-\mathrm{MAA}$ which make more stable of ((CdSe)ZnSe)ZnS) QDs aqueous dispersion as compared then aqua-((CdSe)ZnSe)ZnS)-MAA solution. The fact was identical visually (Figure 1). Further the fact was proved by UV-visible spectra which shows comparatively more pick height in aqua-((CdSe)ZnSe)ZnS)-MAA)-surfactants solution then aqua- $(((\mathrm{CdSe}) \mathrm{ZnSe}) \mathrm{ZnS})-\mathrm{MAA}$ with time . The concentration 
calculation from the compared pick height proves that the loss of less amount of QDs in the aqua-((CdSe)ZnSe)ZnS)-MAA)-surfactants solution then the aqua-((CdSe) $\mathrm{ZnSe}) \mathrm{ZnS})-\mathrm{MAA}$ solution. That is an authentic prove of the conclusion that surfactants coating can prevent the surface oxidation of aqueous dispersed ((CdSe) $\mathrm{ZnSe}) \mathrm{ZnS})$ quantum dots thus can give long term aqueous stability as well as to increase the photostability for long time signal image in biological labeling. To prove our conclusion as the preventing the oxidation of QDs core cells in their aqueous dispersion using surface stabilizing agents: a kinetic measurement was done to measure the exact oxidation rate of the QDs aqueous dispersed using the Arrhenious equation of rate law. The oxidation-degradation is shown in Figure 5 with time. It was found that after 90 hours of the UV-light irradiation treatment the oxidation rate is as: Only with $\mathrm{MAA}=20.72 \times 10^{-5} \mathrm{~s}^{-1}$, QD-MAA-SDS $=7 \times 10^{-5} \mathrm{~s}^{-1}$, QD-MAA-SDBS $=10.36 \times 10^{-5} \mathrm{~s}^{-1}$, QDMAA-CTAB $=6.5 \times 10^{-5} \mathrm{~s}^{-1}$ respectively. This kinetic measurement of oxidation rate gives us the prove about the preventing oxidation using surface functionalizing agents.

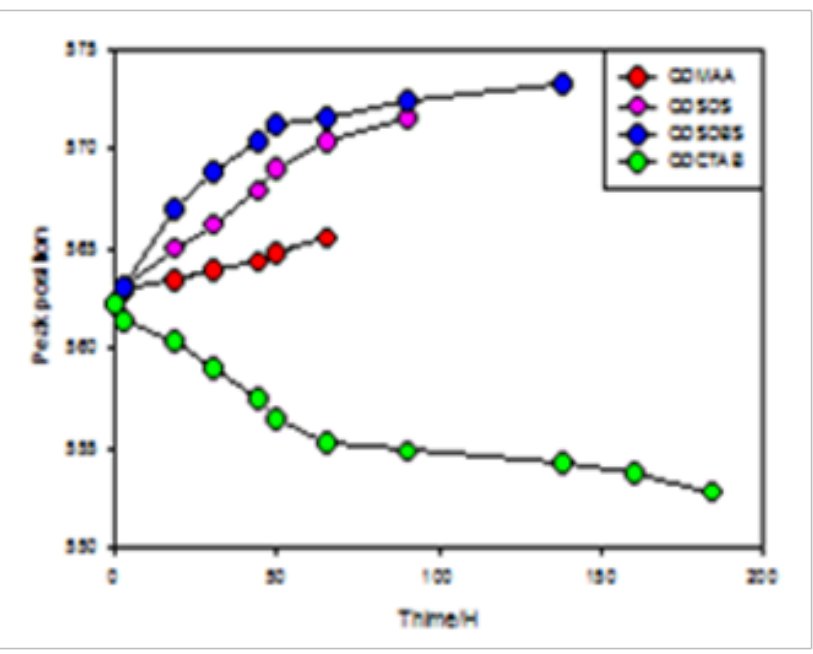

Figure 4 Peak position changes with the progress of oxidation under UV-light irradiation.

Table 3 Change of peak width as the oxidation progress with time

\begin{tabular}{lllll}
\hline Time & Peak width & & & \\
& MAA & SDS & CTAB & SDBS \\
\hline 0 & 24.09 & 24.93 & 24.35 & 24.81 \\
3 & 25.14 & 25.32 & 24.89 & 26.31 \\
18 & 27.40 & 27.69 & 26.17 & 27.63 \\
30 & 27.47 & 28.17 & 26.48 & 27.90 \\
44 & 27.62 & 28.86 & 26.80 & 28.53 \\
50 & 28.46 & 29.21 & 26.98 & 28.77 \\
65 & 29.51 & 30.41 & 27.44 & 28.94 \\
90 & - & 31.98 & 28.97 & 29.16 \\
138 & - & - & 29.38 & 30.93 \\
160 & - & - & 29.98 & - \\
184 & - & - & & - \\
\hline
\end{tabular}

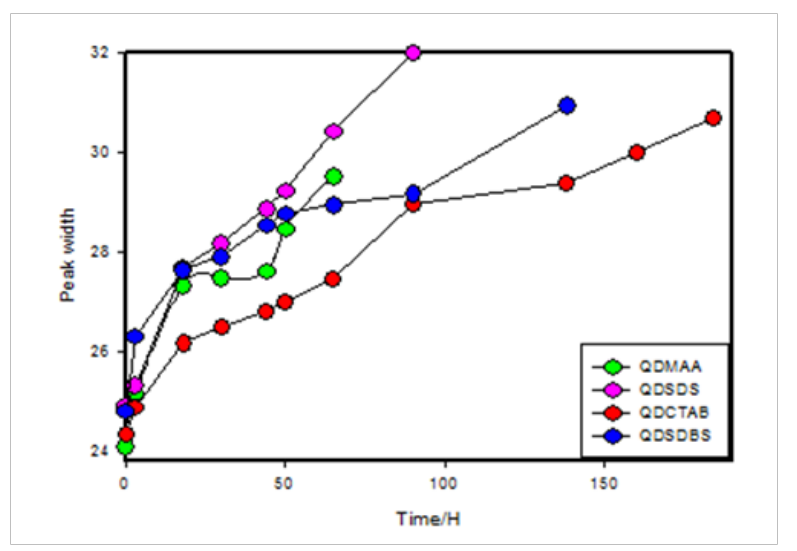

Figure 5 Peak width changes with time is the indication of progress oxidation weakening the bonding energy between the QDs and functionalized molecules.

\section{Toxicity/biocompatibility analysis}

Since constituents of colloidal quantum dots such as cadmium ${ }^{24}$ or selenium ${ }^{25}$ are toxic to many cells, harmful effects can be expected, especially when the hydrophilic shell around the quantum dots is not stable and they might dissolve under the release of toxic ions. In this point of view our result contribute in the stability of quantum dots aqueous dispersion for a long period of time will facilate the less toxicity for the biological cell labeling as compared than the previous less stable aqueous dispersed quantum dots.

\section{Conclusion}

The important findings from this work is that different surfactants can improve the life time of monodispersed quantum dots(QD) in aqueous media preventing the rate of oxidation which breaks the dispersion ability( the electrostatic interaction between aqueous solvent and QD's coating outer surface) and location of the deposition are dependent on the surface coating of the quantum dots that can prevent surface oxidation at air/water interface of aqueous dispersed quantum dots by adhering the quantum dots core cells resulting the long time stability in aqueous even under high UV irradiation ( Figure $6){ }^{26}$
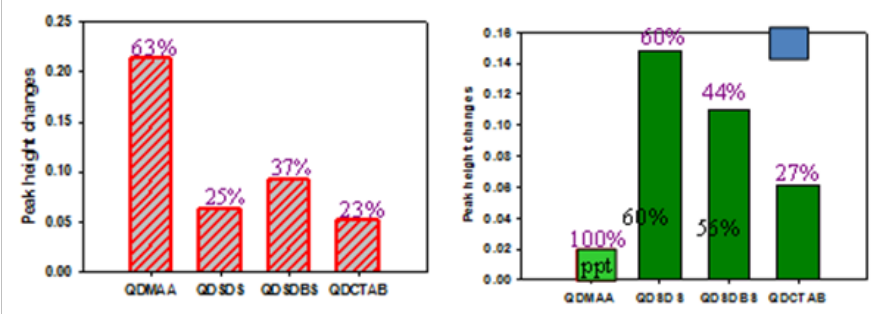

Figure 6 Showing the comparative oxidation rate with different surface coating agents thus the indicating the luminescence lifetime instability with time, (A) after $65 \mathrm{H}$, and (B) After $90 \mathrm{H}$.

\section{Acknowledgements}

Author pays sincere thanks to Nanoscale Characterization and Environmental Chemistry Laboratory, Hanyang University, Seoul, Korea for the instrumental support and to professor Tae-Hyun Yoon for his valuable suggestions for the work. Author also pays sincere thanks to the authority of BK-21 scholarship for their financial support for the work. 


\section{Conflict of interest}

Authors declare that there is no conflict of interest.

\section{References}

1. Bruchez Jr M, Moronne M, Gin P, et al. Semiconductor nanocrystals as fluorescent biological labels. Science. 1998;281:2013.

2. Chan WCW, Nie S. Quantum dot bioconjugates for ultrasensitive nonisotopic detection. Science.1998;281:2016.

3. Murray CB, Norris DJ, Bawendi MG. Synthesis and characterization of nearly monodisperse $\mathrm{CdE}(\mathrm{E}=$ sulfur, selenium, tellurium) semiconductor nanocrystallites. J Am Chem Soc. 1993;115:8706.

4. Hines MA, Guyot-Sionnest P. Synthesis and Characterization of Strongly Luminescing ZnS-Capped CdSe Nanocrystals. J Phys Chem. 1996;100:468.

5. Mitchell GP, Mirkin CA, Letsinger RL. Programmed Assembly of DNA Functionalized Quantum Dots. J Am Chem Soc. 1999;121:8122.

6. Mattoussi H, Mauro JM, Goldman ER, et al. Self-Assembly of CdSe-ZnS Quantum Dot Bioconjugates Using an Engineered Recombinant Protein. J Am Chem Soc. 2000;122:12142.

7. Pathak S, Choi SK, Arnheim N, et al. Hydroxylated quantum dots as luminescent probes for in situ hybridization. $J$ Am Chem Soc. 2001:123:4103.

8. Gerion D, Pinaud F, Williams SC, et al. Synthesis and Properties of Biocompatible Water-Soluble Silica-Coated CdSe/ZnS Semiconductor Quantum Dots. J Phys Chem B. 2001;105: 8861.

9. Wu X, Liu H, Liu J, et al. Immunofluorescent labeling of cancer marker Her2 and other cellular targets with semiconductor quantum dots. Nature Biotechnol. 2003;21:41-46.

10. Larson DR, Zipfel WR, Williams RM, et al. Single Molecule Applications of Quantum Dots. Science. 2003;300:1434.

11. Dubertret B, Skourides P, Norris DJ, et al. In vivo imaging of quantum dots encapsulated in phospholipid micelles. Science. 2002;298:1759.

12. Chan WCW, Nieb SM. Quantum Dot Bioconjugates for Ultrasensitive Nonisotopic Detection. Science. 1998;281:2016.

13. Cordero SR, Carson PJ, Estabrook RA, et al. Photo-Activated Luminescence of CdSe Quantum Dot Monolayers. J Phys Chem B. 2000;104:12137.
14. Coe S, Woo WK, Bawendi M, et al. Electroluminescence from single monolayers of nanocrystals in molecular organic devices. Nature. 2002; $420: 800$.

15. Aldana,Wang YA, Peng XG. Photochemical Instability of CdSe Nanocrystals Coated by Hydrophilic Thiols. $J$ Am Chem Soc. 2001;123:8844.

16. Wong MS, Stucky GD. Am Chem Soc. 2001:221.

17. Kloepfer JA, Bradforth SE, Nadeau JL. Photophysical properties of biologically compatible CdSe quantum dot structures. J Phys Chem B. 2005;109:1996.

18. Schmelz O, Mews A, Basche T, et al. Supramolecular Complexes from CdSe Nanocrystals and Organic Fluorophors. Langmuir. 2001;17:2861.

19. Dabbousi BO, Rodriguezviejo J, Mikulec FV, et al. (CdSe)ZnS CoreShell Quantum Dots: Synthesis and Characterization of a Size Series of Highly Luminescent Nanocrystallites. J Phys Chem B. 1997;101:9463.

20. Wuister SF, Donega CDM, Meijerink A. Luminescence Temperature Antiquenching of Water-Soluble CdTe Quantum Dots: Role of the Solvent. J Am Chem Soc. 2004;126:10397.

21. Spanhel L, Haase M, Weller H, et al. Photochemistry of colloidal semiconductors. 20. Surface modification and stability of strong luminescing CdS particles. J Am Chem Soc. 1987;109:5649.

22. Katari JEB, Colvin VL, Alivisatos AP. X-ray Photoelectron Spectroscopy of CdSe Nanocrystals with Applications to Studies of the Nanocrystal Surface. J Phys Chem. 1994;98:4109.

23. Gao X, Chan WC, Nie S. Quantum-dot nanocrystals for ultrasensitive biological labeling and multicolor optical encoding. J Biomed Opt. 2002; 7:532-537.

24. Kondoh M, Araragi S, Sato K, et al. Cadmium induces apoptosis partly via caspase-9 activation in HL-60 cells. Toxicology. 2002;170:111-117.

25. Shen HM, Yang CF, Ong CN. Sodium selenite-induced oxidative stress and apoptosis in human hepatoma HepG2 cells. J Cance. 1999;81:820 828.

26. Jaiswal JK, Mattoussi H, Mauro JM, et al. Long-term multiple color imaging of live cells using quantum dot bioconjugates. Nature Biotechnol. $2003 ; 21: 47-51$ 